\title{
Stereographic X-ray Reflection Topography of Dislocations in Zinc
}

\author{
By T. VREELAND JR \\ W. M. Keck Laboratory of Engineering Materials, California Institute of Technology, \\ Pasadena, California 91125, U.S.A.
}

(Received 14 May 1975; accepted 24 August 1975)

\begin{abstract}
X-ray reflection topographs were taken of a zinc surface oriented about $1^{\circ}$ from a basal plane. Basal dislocations are revealed in the topographs, and their apparent depth was determined using stereo pairs of topographs. The apparent depths observed in a complimentary pair of topographs using $10 \overline{1} 3$ and $\overline{1} 013$ reflections were significantly greater than those observed in an asymmetric pair of topographs in which the same $10 \overline{1} 3$ reflection was used. This difference is attributed to shifts of the image with respect to the dislocation position. Quantitative estimates of the image shifts and the actual depths of the observed dislocations are obtained from the measurement of apparent depths. Dislocations are visible over the range of depths from 1.7 to $4.5 \mu \mathrm{m}$.
\end{abstract}

\section{Introduction}

The depth of dislocations below the crystal surface, revealed in X-ray reflection topographs of zinc, has been estimated to be about $2.5 \mu \mathrm{m}$ by measuring their projected length when the dislocations intersect the surface at a known small angle (Pope \& Vreeland, 1969). The present investigation was undertaken to check this estimation, and to develop a method whereby the depth of isolated basal dislocations below an (0001) surface could be measured.

Methods for obtaining three-dimensional information from transmission topographs have been discussed by Lang $(1959 a, b)$ and Haruta (1965). Lang's method utilizes topographs taken of the reflection $h k l$ and its inverse $\bar{h} k l$. The stereo 'convergence' angle in binocular vision is then twice the Bragg angle. An exact analog of this method is not possible with reflection topographs, but complimentary topographs $h k l$ and $\bar{h} k l$ may be used giving a stereo angle of $\left(180^{\circ}-2 \theta-\varphi\right)$ where $\theta$ is the Bragg angle and $\varphi$ is the angle between the reflecting planes. Haruta obtained stereo pairs of transmission topographs, using a single Bragg reflection, by rotating the crystal about the normal $\mathbf{g}$ to the diffracting plane between the two topographs. This method is applicable to reflection topographs as well, and has the advantage that the stereo angle may be chosen at will rather than being dictated by the crystal structure.

X-ray reflection topographs usually provide geometrically distorted images because it is seldom practical to align the plate or film parallel to the surface of the crystal (Newkirk, 1959; Turner, Vreeland \& Pope, 1968). This geometric distortion is different in the two members of a stereo pair of reflection topographs obtained by Haruta's method. The distortion must be removed before depth information can be obtained by stereoscopic techniques. We accomplish this in the present investigation by computer image processing of the topographs.

We will compare the results of measurements of dislocation depth made from a complimentary pair of reflections and from a pair of topographs using the same reflection.

\section{Experimental method}

A cylindrical zinc crystal, $0.95 \mathrm{~cm}$ diameter, with its axis parallel to [0001] was prepared by acid machining using a South Bay Technology Model 452 lathing instrument. End surfaces of the cylinder were cleaved in liquid $\mathrm{N}_{2}$ to establish accurately the crystal orientation. The crystal was sheared to produce a small amount of basal slip. One end surface of the crystal was mounted to a goniometer employed in an acid lapping machine (South Bay Technology, Model 451). The other end surface of the crystal was then lapped to a plane oriented $1 \cdot 0 \pm 0 \cdot 1^{\circ}$ from $(0001)$. The maximum slope of the lapped end surface was in the [0110] direction. A square grid pattern of $0 \cdot 1 \mu \mathrm{m}$ thick, $40 \mu$ m diameter gold dots was vacuum evaporated onto the lapped surface to serve as a depth reference.

The topographs used in this study each required a $12 \mathrm{~h}$ exposure, and four topographs were taken consecutively over a $50 \mathrm{~h}$ period. The Berg-Barrett camera system described by Turner, Vreeland \& Pope (1968) was used to record the topographs on Kodak highresolution plates and $\mathrm{Co} K \alpha$ radiation was employed. The first two topographs were $10 \overline{1} 3$ and $\overline{1} 013$ zerolayer reflections (a zero-layer reflection is one in which the incident and reflected X-ray beams are in the same plane as the normal to the surface of the crystal). A $20 \times$ enlarged negative of each of these topographs was made, and contact prints on Dupont Cronopaque were prepared for stereo viewing. The second pair of $10 \overline{1} 3$ topographs was taken with the specimen rotated about the surface normal $\pm 20^{\circ}$ from zero layer (asymmetric or skew-plane reflexions), and $20 \times$ enlarged negatives were made.

\section{Experimental results}

A stereo pair of complimentary $10 \overline{1} 3$ and $\overline{1} 013$ topographs is shown in Fig. 1. Short segments of (0001) 

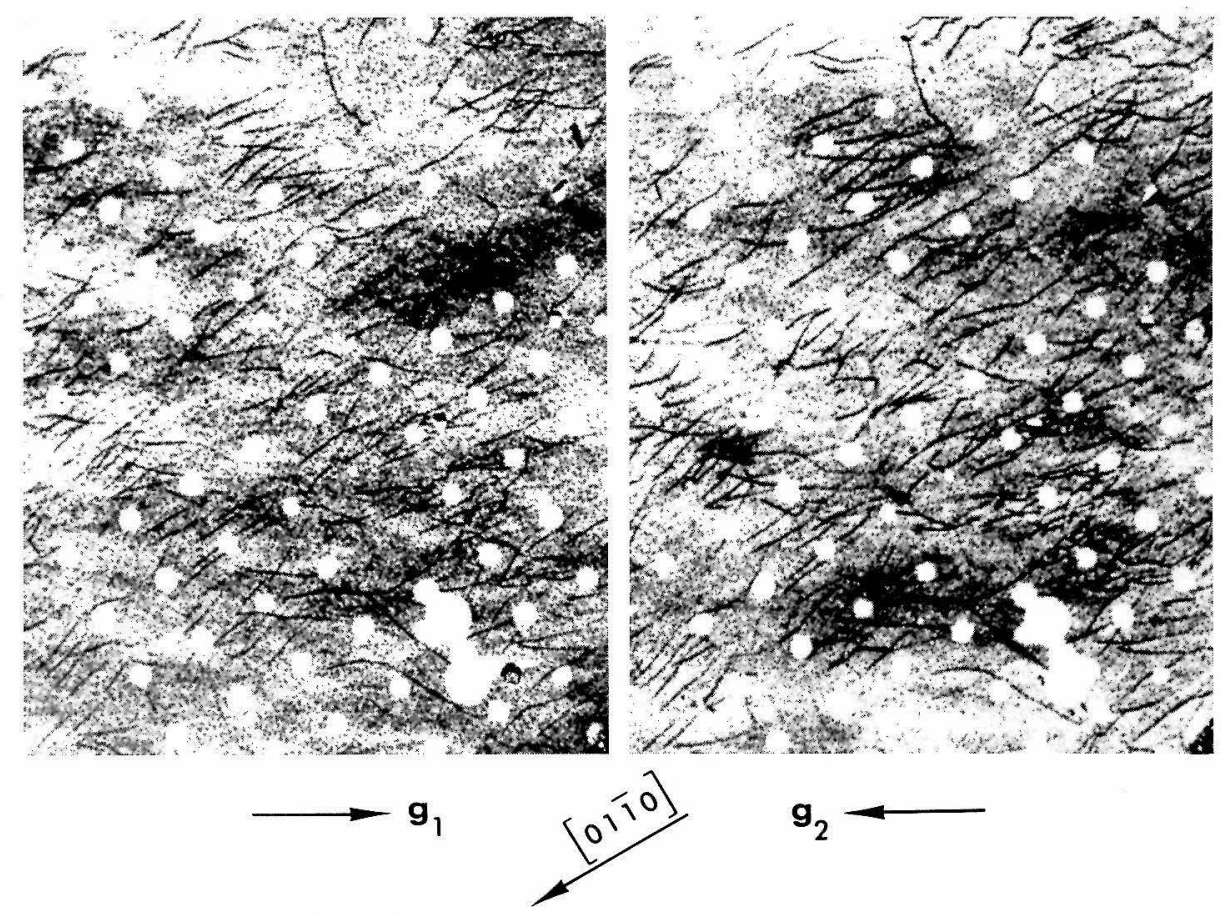

$g_{2} \longrightarrow$

Fig. 1. A stereo pair of complimentary $10 \overline{1} 3$ and $\overline{1} 013$ topographs. A square grid of gold dots, approximately $40 \mu \mathrm{m}$ in diameter, is seen on the surface of the crystal.

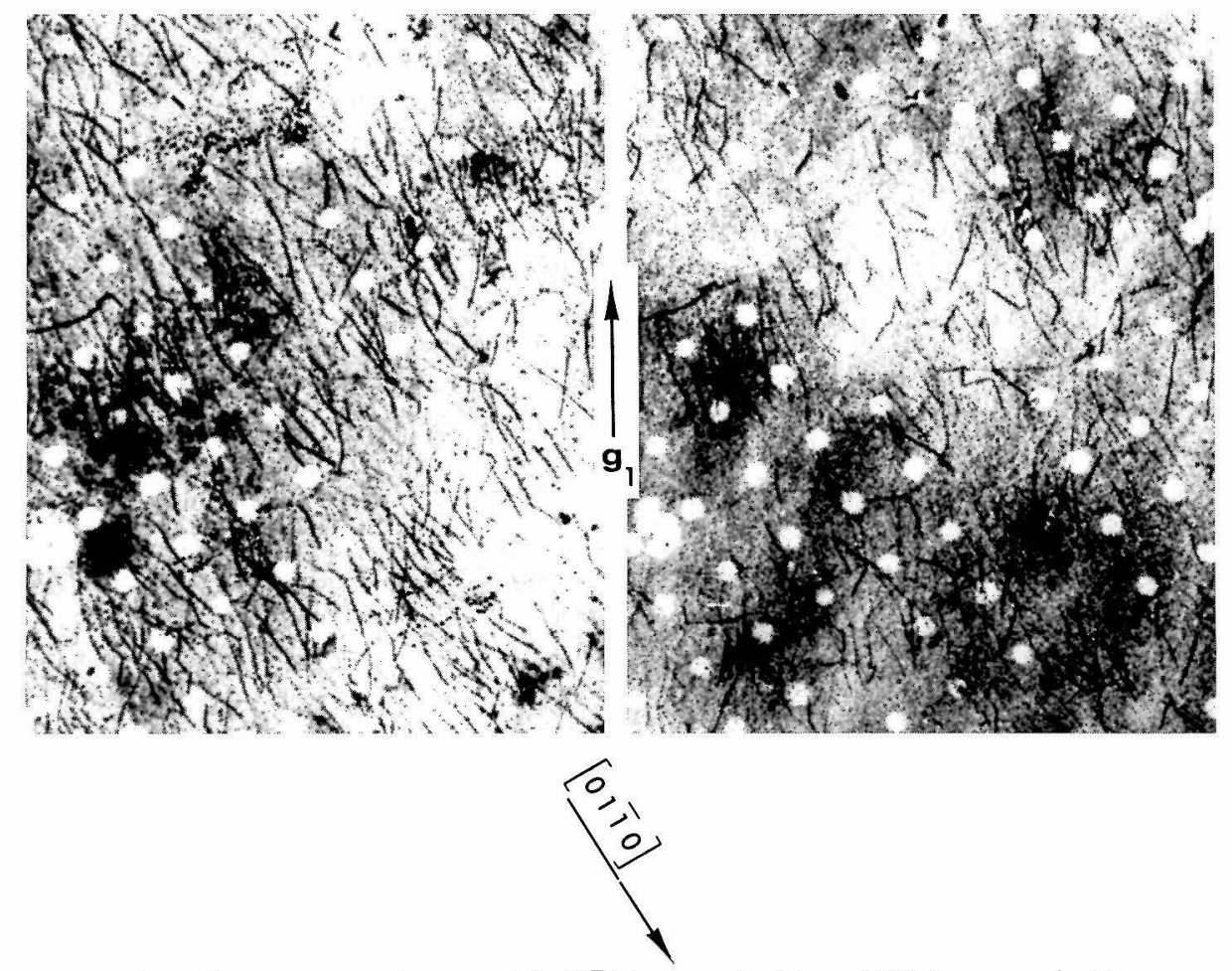

Fig. 2. Image processed asymmetric $10 \overline{1} 3$ topographs taken $\pm 20^{\circ}$ from zero layer. 
[2110] dislocations which extend from the surface into the crystal are revealed. The average projected length of these segments in the [0110] direction is $150 \mu \mathrm{m}$. If we assume that the dislocations have not climbed out of the (0001) slip plane, and that one end of the image corresponds to the surface intersection, we calculate the other end to be $2.6 \mu \mathrm{m}$ deep which is in good agreement with Pope \& Vreeland (1969). The pair of asymmetric topographs rolled $\pm 20^{\circ}$ from zero layer were processed as discussed in the next section.

\section{Computer image processing}

The asymmetric topographs were not suitable for stereo viewing because of the distortions introduced by the X-ray and crystal geometry. These distortions were removed and modifications in the optical density were made using computer image processing (Billingsley, 1971). Selected portions of the enlarged negatives were first digitized. A flying-spot scanner with a spot size of about $35 \mu \mathrm{m}$ was used to digitize the optical density of each spot in an $1500 \times 1500$ array of picture elements, $28 \mu \mathrm{m}$ apart (corresponding to about $1.3 \mu \mathrm{m}$ on the crystal). The optical density of each spot or picture element is characterized by a number corresponding to a gray-level scale which extends from 0 (white) to 255 (black). The gray-scale histograms had mean values of approximately 110 and did not extend to 0 or 255 . In order to enhance the contrast, the extreme ends of the gray scale histograms were set to 0 and 255 respectively and the remaining gray levels were linearly interpolated to cover the full dynamic range.

The distortions introduced by the X-ray geometry were determined by measuring the grid of gold dots on an enlarged topograph, and geometric corrections were applied to the image, which restored the grid to its initial shape. The processed digital data were then converted to photographic negatives (a $22.4 \times$ magnification of the specimen) using a cathode-ray-tube recorder. These negatives were used for the stereoscopic measurements. A stereo pair of processed topographs, photographically enlarged, is shown in Fig. 2. The topographs in Fig. 2 are centered on the same area of the crystal shown in Fig. 1, and they are oriented at $90^{\circ}$ to the topographs of Fig. 1 for stereo viewing.

\section{Stereoscopic measurements}

A mirror stereoscope (Hilger and Watts, Model 5B-180 with binocular unit and 'flying-spot' stereometer) was used to make quantitative measurements of the apparent dislocation depth below the grid of gold dots. The micrometer of the stereometer unit was attached to a ten-turn potentiometer to permit the generation of an analog voltage signal which was proportional to micrometer rotation. The potentiometer was connected in a Wheatstone bridge circuit whose supply voltage could be adjusted to give the desired scale factor be- tween bridge output voltage and micrometer motion (or depth change in the stereo pictures). The bridge output voltage was recorded on a strip-chart recorder when the flying spot of the stereometer was moved between a gold dot on the surface and a dislocation line which appeared below the surface in the stereo pair.

Conversion of the micrometer motion to stereo depth was accomplished by consideration of the X-ray geometry shown in Fig. 3 for the case of complimentary $10 \overline{1} 3$ and $\overline{1} 013$ reflections. For each exposure, the recording film was placed parallel to the incident X-ray beam, $\chi_{1}$, or, $\chi_{2}$, and therefore at an angle $\alpha$ to the crystal surface whose normal is $\mathbf{n}$. A surface point, $a$, maps to $a_{1}$ and $a_{2}$ on films 1 and 2 respectively, while point, $b$, below the surface maps to points $b_{1}$ and $b_{2}$. The stereo convergence angle is $2 \beta_{c}$, and for the Co $K \alpha$ radiation used $\alpha=6.41^{\circ}$ and $\beta_{c}=12.50^{\circ}$ where the subscript $c$ is used to designate the geometry relating to the complimentary pair of topographs. The depth, $h_{c}$, is obtained from the complimentary pair of topographs by use of the relationship,

$$
h_{c}=\frac{\overline{a_{2} b_{2}} \cos \left(\beta_{c}-\alpha\right)}{2 M \sin \beta_{c}}
$$

where $\overline{a_{2} b_{2}}=$ difference in micrometer reading of the flying spot unit between the surface and the dislocation, and

$M=$ the magnification of the films or prints being viewed.

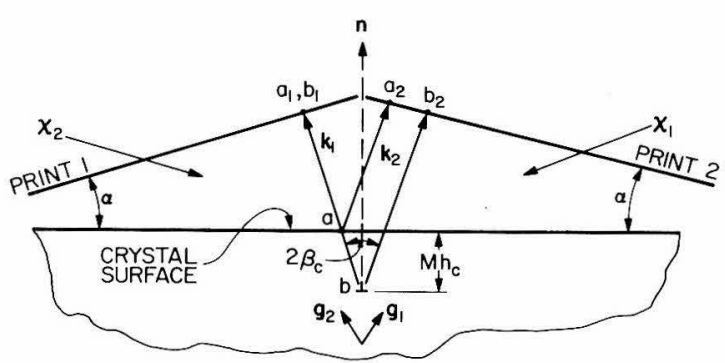

Fig. 3. The geometry for complimentary $10 \overline{1} 3$ and $\overline{1} 013$ reflections. The crystal image has been magnified by the magnification, $M$, of the prints.

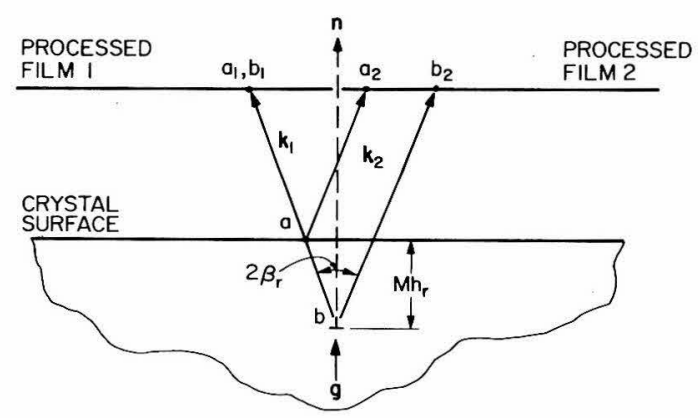

Fig. 4 The stereographic geometry for the image-processed asymmetric 1013 topographs taken $\pm 20^{\circ}$ from zero layer. The crystal image has been magnified by the magnification, $M$, of the films. 
The object-image relationship drawn in Fig. 3 is correct for a mathematical point defect. Defects such as dislocations with a strain field of finite size may form images which are not centered on the $\mathbf{k}$ vector passing through the center of the defect. Equation (1) then only gives an apparent depth. Image shifts are discussed in the next section.

The image-processed topographs (the asymmetric pair $\pm 20^{\circ}$ from zero layer) were analyzed using the stereographic geometry shown in Fig. 4. The apparent dislocation depth is given by

$$
h_{r}=\frac{\overline{a_{2} b_{2}}}{2 M \tan \beta_{r}}
$$

where $\beta_{r}=19 \cdot 7^{\circ}$ for our asymmetric topographs.

Results of the apparent-depth measurements on the stereo pairs of topographs are given in Table 1. Mean values of the measurements made at each end of the segments are listed together with values of the standard deviation and standard error of the mean. The width of the dislocation image is about $3.5 \mu \mathrm{m}$ and the standard deviation of the apparent-depth measurements of a given point was comparable to one image width. The apparent-depth measurements imply dis-

\section{Table 1. Apparent-depth measurements}

$\begin{array}{lccc} & \text { Depth }(\mu \mathrm{m}) & \begin{array}{c}\text { Standard } \\ \text { deviation } \\ (\mu \mathrm{m})\end{array} & \begin{array}{c}\text { Standard } \\ \text { error of the } \\ \text { mean }(\mu \mathrm{m})\end{array} \\ \begin{array}{l}10 \overline{1} 3 \text { and } \overline{1} 013 \text { zero-layer } \\ \text { reflections }\end{array} & 11.6 & 2.5 & 0.52 \\ \quad \begin{array}{l}\text { Shallow end } \\ \text { Deep end }\end{array} & 20.2 & 3.7 & 0.77 \\ \begin{array}{l}\text { Asymmetric } 10 \overline{1} 3 \pm 20^{\circ} \\ \text { from zero layer }\end{array} & & & \\ \quad \begin{array}{l}\text { Shallow end } \\ \text { Deep end }\end{array} & 3.7 & 1.9 & 0.41 \\ & 7.7 & 2.6 & 0.66\end{array}$

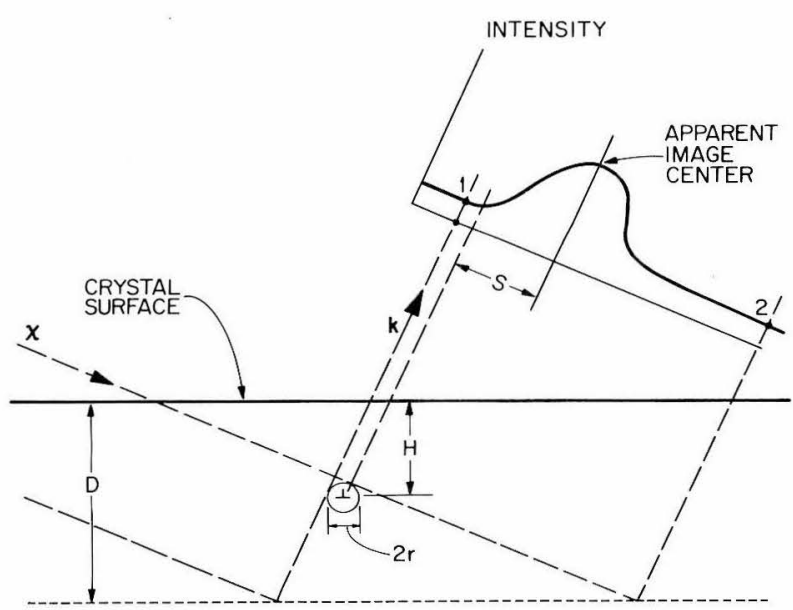

Fig. 5. Schematic of a dislocation-image relationship where an image shift, $S$, takes place. location inclinations from the surface of $3 \cdot 3^{\circ}$ (complimentary pair) and $1.5^{\circ}$ (the asymmetric pair $\pm 20^{\circ}$ from the zero layer), while the inclination must be $1^{\circ}$ for dislocations which lie on the basal plane.

\section{Image shifts}

A possible dislocation-image relationship where an image shift takes place is shown schematically in Fig. 5. Incident X-rays, $\chi$, penetrate to an effective depth, $D$, interact with the dislocation strain field (effective diameter $r$ ), and produce enhanced intensity in the reflection $\mathbf{k}$ between points 1 and 2 . A sketch of the intensity distribution between these points is shown with the apparent image center shifted with respect to the defect center by $S$. (To our knowledge, a calculation of the intensity distribution for the geometry of this experiment has not been made.) Image shifts with the geometry shown in Fig. 5 are equal and opposite in the stereo pair formed using complimentary reflections, and in the direction of $\overline{a_{2} b_{2}}$ (in the plane of $\chi$ and $\mathbf{k}$, Fig. 3). This effect makes the apparent dislocation depth greater than the actual depth.

With the geometry of Figs. 3 and 5, the apparent depth is related to the actual depth, $H_{c}$, and the image shift, $S_{c}$, by the relation

$$
h_{c}=H_{c}+2 S_{c} K_{c}
$$

where $K_{c}=\cos \left(\beta_{c}-\alpha_{c}\right) /\left(2 \sin \beta_{c}\right)$.

Image shifts (in the plane of $\chi$ and $\mathbf{k}$ ) in the stereo pair rolled $\pm 20^{\circ}$ have a component in the direction of $\overline{a_{2} b_{2}}$ (Fig. 4) which also increases the apparent depth, but by a lesser amount than in the complimentary pair of topographs. Since the image shifts are a diffraction contrast effect, they cannot be eliminated in reflection topographs. They can be decreased by decreasing the roll angle, but small roll angles do not give a sufficient stereo effect for quantitative measurements.

We deduce from the experimental geometry that the apparent depth is related to the actual depth $H_{r}$ and the image shift $S_{r}$ in the plane of $\chi$ and $\mathbf{k}$ by the relationship

$$
h_{r}=H_{r}+2 S_{r} K_{r} \sin \beta_{r},
$$

where $K_{r}=1 /\left(2 \tan \beta_{r}\right)$ and use has been made of the relation

$$
\cos \left(\beta_{r}-\alpha_{r}\right) / \cos \alpha_{r} \simeq 1 \text {. }
$$

We must have additional knowledge about the image shifts in order to deduce the actual dislocation depths from our measurements. The observation that the apparent inclination of the dislocations is larger than expected leads us to believe that the image shift increases with dislocation depth (an image shift independent of depth would increase $h$ without changing the inclination). As a first approximation, we will assume that the image shifts in the direction indicated in Fig. 5 (in the plane of $\chi$ and $\mathbf{k}$ ) are given by

$$
S_{c}=A+B H_{c} \quad \text { and } \quad S_{r}=A+B H_{r},
$$


where $A$ and $B$ are constants to be determined from the experimental data.

Comparison of the zero-layer topographs with those rolled $\pm 20^{\circ}$ from the zero layer shows that the shallow and deep ends of the visible dislocation images are not displaced significantly in the direction of the dislocation line. This implies that the actual depth of an end point of a given dislocation in a zero layer and a $\pm 20^{\circ}$ from zero-layer topograph is the same (to within about $0 \cdot 1 \mu \mathrm{m}$ ) or

$$
H_{c}=H_{r} \quad \text { (end point). }
$$

Equations (3)-(6) were used with the data of Table 1 to find $A=1.3 \mu \mathrm{m}$ and $B=0.45$. The actual depths of the shallow and deep ends of the visible dislocations were then calculated and the results are given in Table 2. The difference of $2.8 \mu \mathrm{m}$ between the shallow and deep ends of the dislocations together with the projected image length $(150 \mu \mathrm{m})$ implies an inclination of $1.1^{\circ}$ to the surface. This agrees well with the inclination of the basal plane to the crystal surface, and gives support to the use of the relationship between image shift and dislocation depth given by equation (5).

Table 2. Calculated dislocation depths and a comparison of the apparent and calculated depths

\begin{tabular}{lccc} 
& & \multicolumn{2}{c}{$\begin{array}{c}\text { Apparent depth/ } \\
\text { calculated depth }\end{array}$} \\
& $\begin{array}{c}\text { Calculated } \\
\text { depth }(\mu \mathrm{m})\end{array}$ & $\begin{array}{c}\text { Compli- } \\
\text { mentary pair }\end{array}$ & $\pm 20^{\circ}$ from \\
zero layer
\end{tabular}

Dislocations are not revealed in the topographs within $1.7 \mu \mathrm{m}$ of the crystal surface. With Uragami's (1969) formula, the extinction depth for the $10 \overline{1} 3$ reflection from a (0001) surface using Co $K \alpha$ radiation was calculated to be $1.5 \mu \mathrm{m}$.

It should be emphasized here that the use of equation (5) has been justified on the basis of the experimental measurements alone. While an image shift which increases with dislocation depth will reconcile the various observations of this study, it remains to be shown how such a shift arises. To do this, the dynamical theory of X-ray diffraction in the Bragg geometry must be applied to the case of a dislocation near a free surface. [For a review see Azaroff, Kaplow, Kato, Weiss, Wilson \& Young (1974).] Changes in the displacement field as the dislocation approaches the free surface must play a significant role in the changes of image shift with dislocation depth.

The ratios of apparent depth to calculated depth given in Table 2 show that image shifts cause the apparent depths to increase by a factor which varies from about two (for the asymmetric $\pm 20^{\circ}$ from zerolayer pair) to about seven (for the shallow end of the dislocations in the complimentary pair).

Image shifts which occur in the Bragg geometry were considered in this study. Image shifts may also occur in the Laue geometry and they will give rise to errors in depth measurements. It should be noted that these errors are not eliminated when the same $\mathbf{g}$ is used for both topographs of a stereo pair. For example, image shifts will introduce an error into depth measurements made from stereo pairs in transmission electron microscopy. The error will be much less than that observed in this study, however, because much smaller stereo convergence angles are used in transmission electron stereography.

\section{Conclusions}

1. The apparent depth of dislocations determined by stereo reflection topographs is sensitive to the diffraction used.

2. Image shifts can produce significant differences between apparent and actual dislocation depths.

3. The image shifts and actual depths may be estimated from two sets of stereo topographs (taken under conditions which produce different shifts).

This work was sponsored by the National Science Foundation and by a grant from the President's Fund of the California Institute of Technology. The work of A. Illig on specimen preparation and photography is gratefully acknowledged. The author expresses his thanks to R. H. Selzer and S. Harami of the Jet Propulsion Laboratory for the image-processing work of this study, and to Professor R. E. Villagrana for many helpful discussions throughout the course of this work. Mr. Lawrence Tu's assistance during the initial stages of this work is also gratefully acknowledged.

\section{References}

Azaroff, L. V., Kaplow, R., Kato, N., Weiss, R. J., Wilson, A. J. C. \& Young, R. A. (1974). X-Ray Diffraction. McGraw-Hill.

Billingsley, F. C. (1971). Advanc. Opt. Electron Microsc. 4, 127-159.

Haruta, K. (1965). J. Appl. Phys. 36, 1789-1790.

LANG, A. R. (1959a). Acta Cryst. 12, 249-250.

Lang, A. R. (1959b). J. Appl. Phys. 30, 1748-1755.

Newkir K, J. B. (1959). Trans. AIME, 215, $483-497$.

Pope, D. P. \& VReEland, T. JR (1969). Trans. TMS-AIME, 245, 2447-2449.

Turner, A. P. L., Vreeland, T. JR \& Pope, D. P. (1968). Acta Cryst. A 24, 452-458.

Uragami, T. S. (1969). J. Phys. Soc. Japan, 27, 147-154. 